\title{
Comportamento de busca por serviços de saúde para a detecção da tuberculose
}

Search behavior for health services for the detection of tuberculosis

Comportamiento de búsqueda por los servicios de salud para la detección de la tuberculosis

\section{Jéssica Oliveira Tomberg', Lílian Moura de Lima Spagnolo II, Jenifer Härter ${ }^{I I I}$, Martina Dias da Rosa Martins ${ }^{\mathrm{IV}}$, Roxana Isabel Cardozo Gonzales v}

\begin{abstract}
Resumo: Objetivo: investigar a detecção da tuberculose pulmonar a partir do comportamento de busca por serviços de saúde em municípios do Rio Grande do Sul. Métodos: estudo transversal descritivo, realizado entre 2013 e 2014, mediante entrevista com 290 pessoas com tuberculose pulmonar de municípios da região Sul do país. $\mathrm{Na}$ análise dos dados, utilizou-se estatística descritiva, construindo-se fluxos de comportamento de busca do diagnóstico da doença. Resultados: os serviços especializados foram utilizados por 39,7\% (115) das pessoas para o primeiro atendimento, ofertando baciloscopia de escarro para 64,1\% (186), radiografia de tórax para 57,2\% (166) e testagem para vírus da imunodeficiência humana para 62,7\% (182). Verificou-se que 37,5\% (109) foram diagnosticadas no primeiro serviço de saúde procurado e 62,4\% (181) buscaram outros serviços. Conclusão: a detecção da tuberculose pulmonar se dá, prioritariamente, nos serviços especializados, seguidos pelas unidades de pronto atendimento e pelas unidades de Atenção Primária à Saúde.

Descritores: Tuberculose; Acesso aos Serviços de Saúde; Qualidade, Acesso e Avaliação da Assistência à Saúde; Atenção Primária à Saúde; Saúde Pública
\end{abstract}

Abstract: Objective: to investigate the detection of pulmonary tuberculosis from the search behavior for health services in towns located in Rio Grande do Sul. Methods: cross-sectional and descriptive study conducted between 2013 and 2014, through interviews with 290 people with pulmonary tuberculosis in towns of the south region of the country. When analyzing data, we used descriptive statistics, designing search behavior flows in search of the diagnosis of the disease. Results: specialized services were used by $39.7 \%$ (115) of people for the first visit, offering sputum smear microscopy to $64.1 \%$ (186), chest X-ray to $57.2 \%$ (166) and testing for the human immunodeficiency

\footnotetext{
I Enfermeira. Doutora em Enfermagem. Servidora da Prefeitura Municipal de Pelotas, Pelotas, RS, Brasil. e-mail: jessicatomberg@hotmail.com. ORCID: https://orcid.org/0000-0002-1194-9970

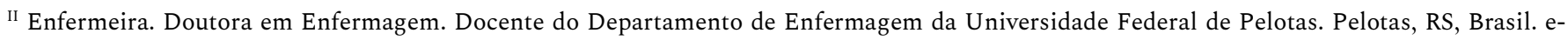
mail: lima.lilian@gmail.com. ORCID: https://orcid.org/0000-0003-2070-6177.

III Enfermeira. Doutora em Enfermagem. Docente do Departamento de Enfermagem da Universidade Federal de Pampa. Uruguaiana, RS, Brasil. e-mail: jeniferharter@hotmail.com. ORCID: https://orcid.org/0000-0002-9130-4290.

IV Acadêmica de Enfermagem da Universidade Federal de Pelotas. Pelotas, RS, Brasil. e-mail: martinadrm@hotmail.com. ORCID: https://orcid.org/0000-0002-8835-6284.

v Enfermeira. Pós Doutora em Enfermagem em Saúde Pública. Docente da Faculdade de Enfermagem da Universidade Federal de Goiás. Goiânia, GO, Brasil. e-mail: roxana_cardozo@hotmail.com. ORCID: https://orcid.org/0000-0001-7180-897X
} 
virus to $62.7 \%$ (182). We found that $37.5 \%$ (109) were diagnosed in the first health service sought and $62.4 \%(181)$ sought other services. Conclusion: the detection of pulmonary tuberculosis takes place, primarily, in specialized services, followed by the Emergency Care Units and the Primary Health Care Units.

Descriptors: Tuberculosis; Health Services Accessibility; Health Care Quality, Access, and Evaluation; Primary Health Care; Public health

Resumen: Objetivo: investigar la detección de la tuberculosis pulmonar basándose en el comportamiento de búsqueda por servicios de salud en ayuntamientos de Rio Grande do Sul. Métodos: estudio transversal descriptivo, realizado entre 2013 y 2014, mediante entrevistas con 290 personas con tuberculosis pulmonar en ayuntamientos de la región sur del país. En el análisis de los datos, se utilizó estadística descriptiva, construyendo flujos de comportamiento de búsqueda del diagnóstico de la enfermedad. Resultados: los servicios especializados se utilizaron por el 39,7\% (115) de las personas para la primera atención, ofreciendo microscopía de esputo al 64,1\% (186), radiografía de tórax al 57,2\% (166) y pruebas de detección del virus de la inmunodeficiencia humana al 62.7\% (182). Se encontró que el 37,5\% (109) fueron diagnosticados en el primer servicio de salud buscado y el 62,4\% (181) buscaron otros servicios. Conclusión: la detección de la tuberculosis pulmonar ocurre, prioritariamente, en los servicios especializados, seguidos por las Unidades de Pronta Atención y las unidades de Atención Primaria de Salud.

Descriptores: Tuberculosis; Accesibilidad a los Servicios de Salud; Calidad, Acceso y Evaluación de la Atención de Salud; Atención Primaria de Salud; Salud Pública

\section{Introdução}

A tuberculose faz parte da agenda de prioridades de políticas públicas de saúde da Organização Mundial da Saúde (OMS) por se caracterizar como uma condição crônica, infectocontagiosa e com altas taxas de mortalidade. No ano de 2015, a OMS aprovou a estratégia “End TB”, que tem como objetivo a eliminação da tuberculose como problema de saúde pública (<1 caso por 100.000 habitantes) e a redução de $95 \%$ das mortes por tuberculose até $2035 .{ }^{1}$

O Brasil compõe o grupo de países que concentram $50 \%$ dos casos de tuberculose no mundo. Em 2018, o país apresentou uma taxa de incidência de 34,8 casos por 100 mil habitantes e taxa de mortalidade em 2017 de 2,18 óbitos por 100 mil habitantes. ${ }^{2-3}$ Para reduzir esses indicadores e alinhar as ações de controle com as metas propostas pela OMS, o Programa Nacional de Controle da Tuberculose (PNCT) definiu como um dos pilares de ação a prevenção 
3 | Tomberg JO, Spagnolo LML, Härter J, Martins MDR, Gonzales RIC

e o cuidado centrados na pessoa com tuberculose, tendo como objetivo diagnosticar precocemente todas as formas de doença. ${ }^{3-4}$

Nesse sentido, é indispensável a detecção precoce do sintomático respiratório (pessoas com tosse há três semanas ou mais), principalmente na Atenção Primária à Saúde (APS), a qual, devido ao seu potencial de localização geográfica e processo de trabalho, tem a responsabilidade na organização e coordenação da assistência aos usuários. Contudo, a atenção à tuberculose é compromisso de todos os serviços de saúde. ${ }^{3-5}$ Por isso, é necessário que trabalhem de forma integrada e possuam fluxos preestabelecidos e apoio laboratorial.

A detecção da tuberculose é estabelecida quando há adequação entre a oferta dos serviços e as necessidades em saúde dos indivíduos, envolvendo aspectos dos usuários, incluindo características econômicas, sociais e culturais, bem como características de ordem estrutural, organizacional e de gestão das unidades de saúde. ${ }^{6-10}$ Pesquisas operacionais realizadas no Brasil e em nível internacional apontam dificuldades de acesso dos sintomáticos respiratórios à detecção precoce da tuberculose. ${ }^{7-11}$ Há evidências da não resolutividade do caso no primeiro serviço de saúde procurado, sendo esta influenciada pela modalidade de atendimento do local. Essa situação determina o comportamento de busca das pessoas por atendimento e o atraso no diagnóstico da doença. ${ }^{7}$

Diante dessas considerações, torna-se relevante conhecer o comportamento de busca ao diagnóstico da tuberculose a partir dos serviços de saúde procurados pelas pessoas com sintomas respiratórios da doença em municípios do Rio Grande do Sul. Isso será essencial para conhecer as potencialidades e fragilidades da atenção à tuberculose, identificando os serviços que fazem o diagnóstico no primeiro contato do usuário. Cabe considerar que pouco se sabe sobre o comportamento de busca dos sintomáticos respiratórios no processo de detecção da tuberculose no estado do Rio Grande do Sul. Como questão de pesquisa tem-se: como se dá a 
busca por atendimento dos sintomáticos respiratórios de tuberculose em municípios do Rio Grande do Sul?

Assim, o presente estudo objetivou investigar a detecção da tuberculose pulmonar a partir do comportamento de busca por serviços de saúde em municípios do Rio Grande do Sul.

\section{Método}

Estudo transversal descritivo, realizado a partir uma pesquisa multicêntrica, a qual teve a coleta de dados com duração de 12 meses, ocorrendo entre 2013 e 2014 nos municípios de Canoas, Pelotas, Santa Cruz do Sul e Sapucaia do Sul, localizados no estado do Rio Grande do Sul. Esses municípios foram selecionados por fazerem parte da lista de municípios prioritários para o controle da tuberculose no Rio Grande do Sul e possuírem a municipalização do PNCT. Ademais, integravam o estudo multicêntrico em parceria com instituições de ensino superior de cada município. Quanto aos dados da tuberculose, em 2014, o município de Canoas notificou 179 casos novos de tuberculose pulmonar, Pelotas 141, Sapucaia do Sul 61 e Santa Cruz do Sul 42.12-13

A população foi composta por todos os indivíduos com tuberculose pulmonar que iniciaram o tratamento no período de coleta de dados. Aplicaram-se os seguintes critérios de inclusão: ter diagnóstico de tuberculose pulmonar; estar em início de tratamento (até três meses); ter idade igual ou superior a 18 anos; não apresentar dificuldades cognitivas que impossibilitassem a aplicação do formulário; e não estarem institucionalizados.

Para os procedimentos de coleta de dados, primeiramente, identificaram-se as pessoas diagnosticadas com tuberculose durante o período de coleta de dados, por meio da vigilância epidemiológica de cada município, e fez-se a busca destas nos serviços de saúde onde estavam sendo acompanhadas para o tratamento da tuberculose. As entrevistas foram realizadas por alunos de pós-graduação, previamente capacitados, utilizando-se um questionário estruturado, previamente codificado. 
5 | Tomberg JO, Spagnolo LML, Härter J, Martins MDR, Gonzales RIC

As variáveis utilizadas foram: número de vezes que buscou serviços de saúde; tipos de serviços de saúde procurados até o diagnóstico da tuberculose; serviço de saúde que diagnosticou a doença; e o serviço de saúde que solicitou exames (radiografia de tórax, baciloscopia de escarro e anti-HIV devido a elevados índices de infecção por tuberculose em indivíduos HIV positivo relacionados às questões de alteração na imunidade). ${ }^{5}$

Os serviços foram agrupados em: unidades de APS (Unidades Básicas de Saúde, com e sem o Programa de Agentes Comunitários de Saúde, e as unidades com Estratégia de Saúde de Família); serviços especializados (SE) (compreendendo os pontos de atenção secundária e terciária, sendo eles os ambulatórios, clínicas, consultórios e hospitais); e unidades de Pronto Atendimento (PA) (constituídas pelas unidades de urgência e emergência).

Para a análise do presente estudo, excluíram-se da amostra aqueles entrevistados que não haviam informado o primeiro serviço procurado para possibilitar a construção do fluxo de comportamento de busca. Os dados foram inseridos em um banco construído no software Excel da Microsoft $^{\circledR}$ e analisados no software Stata $^{\circledR}$ 13.0, aplicando-se estatística descritiva com distribuição de frequências relativa e absoluta das variáveis supracitadas. Utilizou-se a mediana como medida de tendência central para variáveis sem distribuição normal. A partir dos resultados, construiu-se um fluxograma do comportamento de busca das pessoas por modalidade de serviço até a obtenção do diagnóstico. Para essa construção, utilizaram-se os resultados das variáveis "tipos de serviços de saúde procurados até o diagnóstico da tuberculose" e "serviço de saúde que diagnosticou a doença”, o que permitiu identificar dentre os serviços procurados aquele que realizou o diagnóstico.

No estudo, foram respeitados os preceitos éticos da Resolução 466/12 do Conselho Nacional de Saúde. ${ }^{14}$ O projeto foi submetido à Plataforma Brasil com parecer favorável nº 702.283 em 20 de junho de 2013. A aplicação do formulário estruturado aos participantes 
ocorreu em ambiente privativo, mediante concordância dos entrevistados e a assinatura do Termo de Consentimento Livre e Esclarecido em duas vias.

\section{Resultados}

A amostra resultou em 290 entrevistas analisadas. Do total de entrevistados, observou-se a mediana de duas idas aos serviços de saúde até a obtenção do diagnóstico. Verifica-se que 23,8\% (69) escolheram a APS como o primeiro serviço de saúde procurado diante dos sintomas respiratórios, enquanto 39,7\% (115) buscaram por SE e 36,5\% (106) optaram por iniciar o atendimento a partir das unidades de PA. A APS diagnosticou 16,2\% (47) dos entrevistados, os SE $66,9 \%(194)$ e as unidades de PA $16,9 \%(49)$.

Dentre as 69 pessoas que iniciaram a busca por atendimento na APS (Figura 1), 36,3\% (25/69) receberam o diagnóstico na primeira unidade de APS procurada e 63,7\% (44/69) buscaram um segundo serviço de saúde. Dentre estes 44 que procuraram um segundo serviço, por permanecerem sem o diagnóstico, 72,7\% (32/44) o receberam no segundo serviço. Desse modo, restaram 12 pessoas sem o diagnóstico, as quais buscaram por um terceiro serviço. Destas, 66,6\% (8/12) foram diagnosticadas nessa ocasião, restando ainda 41,6\% (4/12) que efetivaram o diagnóstico no quarto serviço de saúde procurado. 
Figura 1 - Fluxo do comportamento de busca por serviços de saúde até a obtenção do diagnóstico da tuberculose, iniciado a partir da APS como primeira escolha, Rio Grande do Sul, Brasil, $2014(\mathrm{n}=69)$

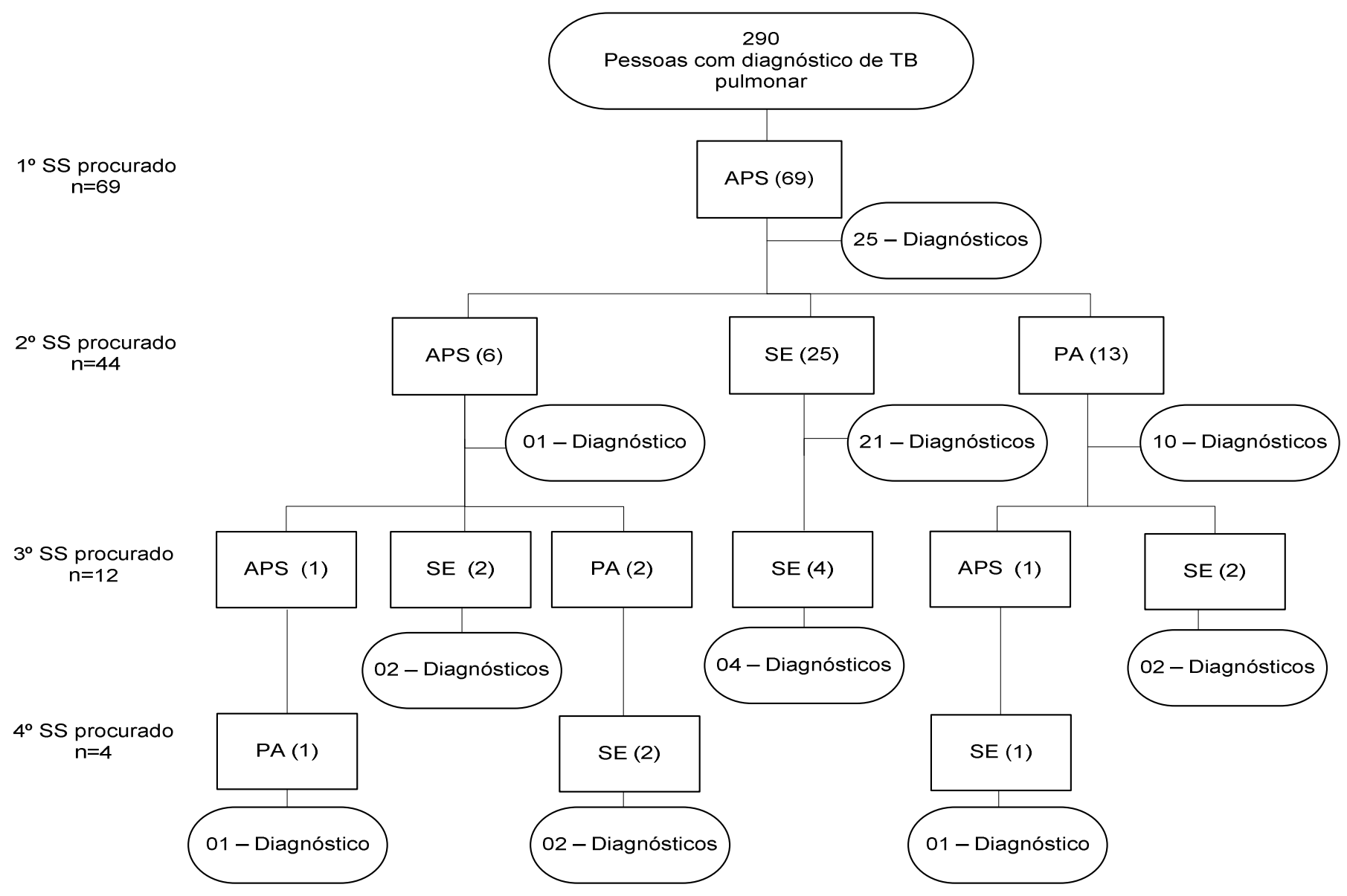

Nota: Serviços de Saúde (SS), Atenção Primária à Saúde (APS), Serviços Especializados (SE), Pronto Atendimento (PA).

Na Figura 2, verifica-se o comportamento de busca de 115 pessoas que optaram pelos SE como primeira opção de atendimento. Destas, foram diagnosticadas $47,0 \%$ (54/115) no primeiro SE procurado e 53,0\% (61/115) buscaram por um segundo serviço de saúde. Das 61 pessoas que procuraram pelo segundo serviço, 65,5\% (40/61) obtiveram o diagnóstico e 34,4\% (21/61) permaneceram sem diagnóstico. A busca pelo terceiro serviço de saúde ocorreu para 21 pessoas, na ocasião $76,1 \%$ (16/21) foram diagnosticadas e 23,8\% (5/21) das pessoas necessitaram realizar a quarta busca por serviço para obter o diagnóstico. 
Figura 2 - Fluxo do comportamento de busca por serviços de saúde até a obtenção do diagnóstico da tuberculose, iniciado a partir dos SE como primeira escolha, Rio Grande do Sul, Brasil, 2014 $(\mathrm{n}=115)$

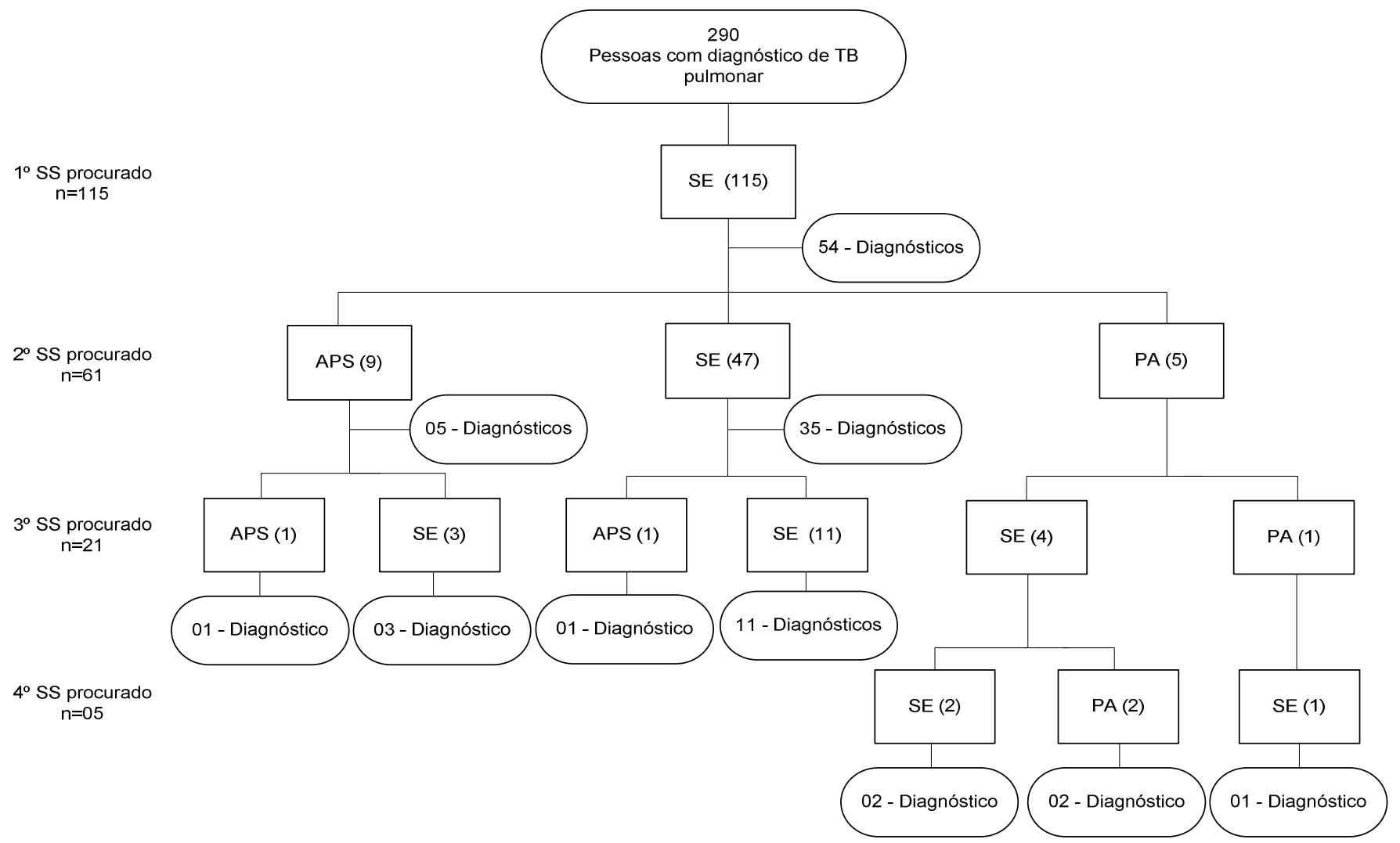

Nota: Serviços de Saúde (SS), Atenção Primária à Saúde (APS), Serviços Especializados (SE), Pronto Atendimento (PA).

Na Figura 3, apresenta-se o fluxo do comportamento de busca de 106 entrevistados, os quais escolheram como primeiro serviço para o atendimento as unidades de PA. Destes, $28,3 \%$ (30/106) obtiveram o diagnóstico no PA após o primeiro atendimento e 71,7\% (76/106) buscaram pelo segundo serviço de saúde. Nessa segunda procura, 67,1\% (51/76) foram diagnosticados, dos quais 76,5\% (39/51) em SE. Das 32,9\% (25/76) pessoas que fizeram uma terceira procura de serviço de saúde em busca do diagnóstico, 76,0\% (19/25) foram diagnosticadas, sendo 73,7\% (14/19) em 
SE. Os 24,0\% (6/25) restantes procuraram um quarto serviço. Nessa ocasião, 50\% (3/6) foram diagnosticadas em SE.

Figura 3 - Fluxo do comportamento de busca por serviços de saúde até a obtenção do diagnóstico da tuberculose, iniciado a partir das unidades de PA como primeira escolha, Rio Grande do Sul, Brasil, $2014(\mathrm{n}=106)$

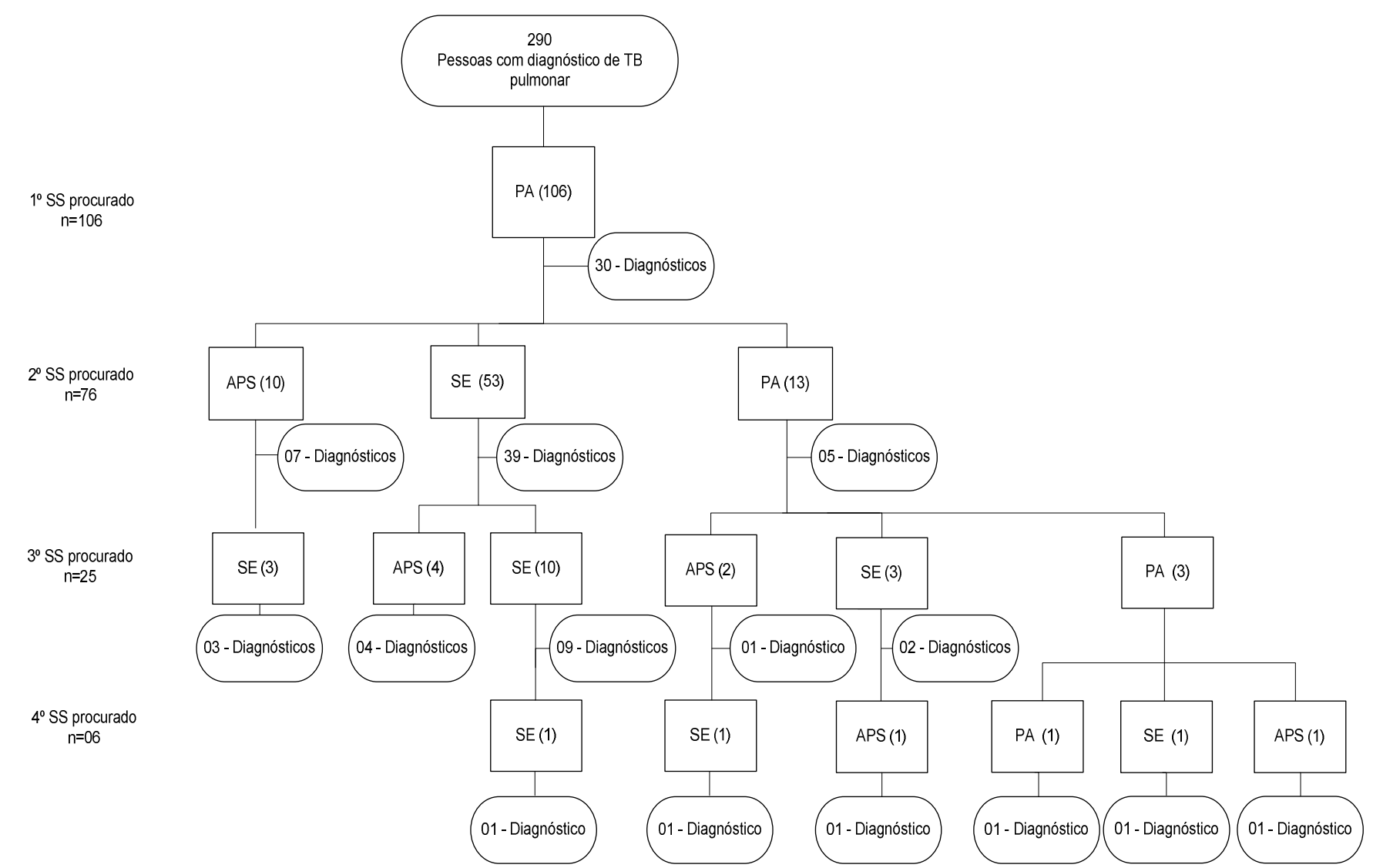

Nota: Serviços de Saúde (SS), Atenção Primária à Saúde (APS), Serviços Especializados (SE), Pronto Atendimento (PA).

$\mathrm{Na}$ Tabela 1, verificam-se os exames ofertados conforme o primeiro serviço de saúde procurado. Considerando os 290 entrevistados, constatou-se que os SE foram responsáveis pela solicitação de 64,1\% (186/290) das baciloscopias de escarro, 57,2\% (166/290) dos pedidos de radiografia de tórax e 62,7\% (182/290) dos testes anti-HIV. 
Tabela 1 - Solicitação de exames e diagnóstico da tuberculose de acordo com o primeiro serviço de saúde procurado, Rio Grande do Sul, Brasil, 2014 (n=290)

\begin{tabular}{|c|c|c|c|c|c|c|c|c|}
\hline \multirow{3}{*}{$\begin{array}{l}\text { Serviços de saúde } \\
\text { que ofertaram os } \\
\text { exames }\end{array}$} & \multicolumn{8}{|c|}{ Primeiro serviço de saúde procurado } \\
\hline & \multicolumn{2}{|c|}{ APS (69) } & \multicolumn{2}{|c|}{ SE (115) } & \multicolumn{2}{|c|}{ PA (106) } & \multicolumn{2}{|c|}{ Total $(290)$} \\
\hline & $\mathrm{n}$ & $\%$ & $\mathrm{n}$ & $\%$ & $\mathrm{n}$ & $\%$ & $\mathrm{n}$ & $\%$ \\
\hline \multicolumn{9}{|l|}{ Baciloscopia de escarro } \\
\hline APS & 40 & 58 & 10 & 8,7 & 12 & 11,3 & 62 & 21,4 \\
\hline SE & 26 & 37,7 & 91 & 79,1 & 69 & 65,1 & 186 & 64,1 \\
\hline PA & 2 & 2,9 & - & - & 17 & 16,1 & 19 & 6,5 \\
\hline Sem & 1 & 1,4 & 14 & 12,2 & 8 & 7,5 & 23 & 8 \\
\hline \multicolumn{9}{|l|}{ Radiografia de tórax } \\
\hline APS & 35 & 50,7 & 7 & 6,1 & 9 & 8,4 & 51 & 17,6 \\
\hline SE & 26 & 37,7 & 98 & 85,2 & 42 & 39,7 & 166 & 57,2 \\
\hline PA & 6 & 8,7 & 3 & 2,6 & 54 & 50,9 & 63 & 21,7 \\
\hline Sem & 2 & 2,9 & 7 & 6,1 & 1 & 1 & 10 & 3,5 \\
\hline \multicolumn{9}{|l|}{ Anti-HIV } \\
\hline APS & 20 & 28,9 & 12 & 10,4 & 14 & 13,2 & 46 & 15,9 \\
\hline SE & 38 & 55 & 77 & 67 & 67 & 63,2 & 182 & 62,7 \\
\hline PA & 2 & 2,9 & - & - & 10 & 9,4 & 12 & 4,1 \\
\hline Sem & 9 & 13,2 & 26 & 22,6 & 15 & 14,1 & 50 & 17,3 \\
\hline
\end{tabular}

Nota: Atenção Primária à Saúde (APS), Serviços Especializados (SE), Pronto Atendimento (PA).

Dentre as 69 pessoas que iniciaram o percurso de busca pelo diagnóstico a partir da APS, percebeu-se que esse nível de atenção foi o responsável por 58,0\% (40/69) das solicitações de baciloscopia de escarro e por 50,7\% (35/69) das solicitações de radiografias de tórax, ficando apenas com 28,9\% (20/69) de solicitações de testes anti-HIV. Em relação aos 106 que buscaram o PA como primeiro serviço para o atendimento, a radiografia de tórax foi o exame, predominantemente, solicitado a 50,9\% (54/106) das pessoas. Para os 115 que iniciaram o atendimento pelos SE, 79,1\% (91/115) tiveram solicitação de baciloscopia de escarro, $85,2 \%$ (98/115) de radiografia de tórax e 67,0\% (77/115) de testes anti-HIV. 


\section{Discussão}

Diante dos resultados da pesquisa, verificaram-se fragilidades na detecção da tuberculose, uma vez que, ao analisar o comportamento de busca, mais da metade das pessoas com tuberculose necessitou procurar mais de um serviço para efetivar o diagnóstico, produzindo fluxos diversificados na rede de serviços de saúde até a confirmação da doença. Reconhece-se que a necessidade de buscar diversos serviços e a modalidade do serviço procurado para o primeiro atendimento são determinantes para o atraso no diagnóstico da doença..$^{7-8}$

Ao analisar o comportamento de busca por atendimento, diante dos primeiros sintomas da doença, os resultados mostram que as unidades de APS foram as menos procuradas entre os entrevistados, sendo os SE os mais procurados para o primeiro atendimento, seguidos pelas unidades de PA. Resultados semelhantes foram identificados em pesquisa realizada no ano de 2010, em 7 municípios brasileiros, e em pesquisa realizada na Etiópia, no ano de 2014, na qual os autores identificaram os SE como os escolhidos para o primeiro atendimento. Em contrapartida, estudo realizado em 2013, nas 26 capitais brasileiras e no Distrito Federal, aponta as unidades de APS como a escolha preferencial para o primeiro atendimento. ${ }^{10,15-16}$ Diante disso, compreende-se que não há um comportamento padrão de busca por atendimento em um determinado serviço de saúde.

Os fatores que limitam a procura pelas unidades de APS foram evidenciados em pesquisa desenvolvida em um país do continente africano, sendo relacionados a não resolutividade das necessidades em saúde e à falta de recursos materiais e humanos. ${ }^{17}$ Igualmente, esses fatores foram apontados em pesquisa realizada no Brasil, somando-se a inexistência de vínculo prévio e as dificuldades na obtenção de consultas e na realização de exames. ${ }^{18}$

Os resultados mostraram que a definição do diagnóstico da tuberculose ocorre majoritariamente em SE, seguido pelas unidades de PA e unidades de APS. Resultados semelhantes foram observados em outras pesquisas apontando fragilidades das unidades de APS para proporcionar diagnóstico oportuno da doença quando se constituem o primeiro serviço 
procurado. ${ }^{19-20}$ Isso atribui-se ao fato de os profissionais atuantes nas unidades de APS não suspeitarem de tuberculose na primeira consulta, não solicitarem o exame de baciloscopia de escarro diante de um sintomático respiratório e se encaminharem para outros serviços. ${ }^{2,11,19}$ Em contrapartida, é possível justificar a baixa detecção da tuberculose das unidades de PA devido à assistência ser centrada no atendimento às situações agudas à saúde, a insuficiência de recursos humanos para o elevado quantitativo de atendimentos e as práticas de educação continuada não serem voltadas para a problemática da tuberculose.

No que tange aos melhores resultados obtidos pelos SE para a detecção da tuberculose, no presente cenário de estudo, acredita-se que estejam também relacionados à forma de organização da atenção à tuberculose, em três dos quatro municípios que integram a pesquisa, os quais são caracterizados pela oferta centralizada do tratamento da tuberculose em ambulatórios do Programa Municipal de Controle da Tuberculose (PMCT), ficando para a APS o papel de desenvolver ações de detecção da doença.

Contudo, vale destacar que o atendimento em tais ambulatórios também contempla o desenvolvimento de ações de detecção de casos. Tanto o diagnóstico quanto o tratamento nesses serviços ocorrem por demanda espontânea ou mediante encaminhamentos. Acredita-se que por possuírem equipes preparadas para atenção direcionada à tuberculose haja a detecção precoce e consequentemente maior busca por atendimento nesses serviços diante de sintomas respiratórios. Ressalta-se ainda que esses serviços de saúde estão dotados de uma estrutura física e de equipamentos apropriados para a agilidade no diagnóstico da doença.

Ademais, os encaminhamentos das unidades de APS para os SE já foram evidenciados como fator resultante do alto quantitativo de buscas por essa modalidade de serviço. ${ }^{15,19}$ Nesse sentido, é importante destacar que os encaminhamentos devem ser norteados por fluxos municipais definidos, que incluam a participação dessas unidades na detecção da tuberculose e na coordenação do cuidado. 
Cabe ressaltar que a tuberculose é uma condição de atendimento sensível à APS por se tratar de uma condição crônica de tratamento prolongado, no mínimo seis meses, que necessita da proximidade do serviço da pessoa doente, no sentido de coordenar a sua assistência. No entanto, a busca por esses serviços é influenciada por aspectos das organizações dos sistemas de saúde, as quais podem facilitar ou limitar, e pela consequente aceitabilidade dos serviços por parte das pessoas com tuberculose. ${ }^{6}$ Verifica-se que, apesar dos esforços governamentais para a consolidação do modelo assistencial a partir da APS, sua efetivação ainda se constitui como um desafio para a gestão em saúde, uma vez que há limitações no acesso, em grande parte relacionada às fragilidades de atributos essenciais dessas unidades. ${ }^{18}$

Nesse sentido, independente do ponto de atenção, ressalta-se o importante papel da gestão dos serviços de saúde em manter as equipes de saúde sempre sensibilizadas para a questão da tuberculose, reconhecendo a doença como um problema de saúde pública e atuando de acordo com as ações programáticas do controle. ${ }^{11,15}$ Para isso, os gestores municipais necessitam valorizar a questão da detecção precoce da tuberculose, fornecendo o suporte para as equipes de saúde no âmbito estrutural, organizacional e operacional. Ratifica-se que fluxos assistenciais sem a inclusão da APS como norteadora no processo de detecção da tuberculose mostram-se ineficientes para o diagnóstico precoce, tal fato tem relação direta com as características de modelo de atenção voltadas para a inserção dos profissionais no ambiente da comunidade, viabilizando as ações de busca ativa. ${ }^{9}$

Com relação à oferta de exames, os SE se destacaram contribuindo com a maior parte das solicitações de baciloscopia de escarro, radiografia de tórax e teste anti-HIV. Dentre os exames, o menos ofertado foi o teste anti-HIV. Nesse contexto, percebe-se que os serviços de saúde não seguem as normas nacionais para o controle da tuberculose, visto que é preconizado que o primeiro serviço procurado, mediante a suspeita da tuberculose, necessita fazer a solicitação imediata da baciloscopia de escarro, da radiografia de tórax e do teste anti-HIV. ${ }^{5}$ Salienta-se que 
desde 2013 os serviços públicos de saúde têm à disposição os kits de testes rápidos para HIV, o que agiliza a realização do exame, sem a necessidade de encaminhamento para a rede laboratorial. ${ }^{5}$

Os resultados obtidos em relação às solicitações de exames, pelo primeiro serviço de saúde procurado, retratam a baixa suspeição das equipes de saúde, no primeiro contato, para a tuberculose, o que gera outras hipóteses diagnósticas, a não oferta dos exames essenciais da tuberculose e o indicativo de outros tratamentos..$^{15,20}$ Tal realidade compromete a interrupção da cadeia de transmissão da doença, onera o sistema de saúde com tratamentos ineficazes e ainda agrava o quadro clínico da pessoa com tuberculose, demandando, muitas vezes, internações hospitalares para manejo das complicações.

Os resultados da presente pesquisa indicam a importância da avaliação da detecção da tuberculose nos serviços de saúde como ferramenta para subsidiar o planejamento das ações de controle da tuberculose, a qual contribui para a discussão na formulação da linha de cuidado da tuberculose, uma vez que apontaram a necessidade da reorganização da atenção à tuberculose, a partir das unidades de APS, e a sua efetivação como coordenadora do cuidado da rede de atenção às pessoas com a doença. Ademais, refletem a necessidade de mudança das práticas em saúde no processo de detecção da tuberculose a partir da sensibilização dos profissionais de saúde para a problemática da doença.

\section{Conclusão}

Os presentes resultados apontam que o comportamento de busca por atendimento nos primeiros sintomas da doença se dá, prioritariamente, por meio dos SE, com 194 diagnósticos, seguidos pelas unidades de PA, com 49 diagnósticos, e pelas unidades de APS, com 47 diagnósticos. Verificou-se ainda a preferência pelos SE, seguidos pelas unidades de PA e por último as unidades de APS. A oferta de exames, essenciais para o diagnóstico da doença, 
ocorreu majoritariamente pelos SE. Considera-se que o fluxo da busca por atendimento nos serviços de saúde até a detecção da tuberculose foi mais dificultoso para aquelas pessoas que optaram pelo primeiro atendimento no PA.

Desse modo, o estudo mostra a necessidade de investir na resolutividade das unidades de APS para torná-las uma opção viável e prioritária na busca por atendimento entre as pessoas com tuberculose. É premente prover os serviços de saúde da qualificação física, funcional e de recursos humanos para ir ao encontro da responsabilidade política assumida pelos gestores para a eliminação da tuberculose como problema de saúde pública no Brasil. A presente pesquisa apresenta como limitação um possível viés de memória relacionado às respostas referentes aos serviços de saúde buscados, aos serviços que solicitaram os exames e aos que diagnosticaram a tuberculose, embora a entrevista tenha sido realizada no início do tratamento.

\section{Referências}

1. World Health Organization (WHO). Stop TB Partnership. Global plan to end TB. The paradigm shift 2016-2020 [Internet]. Geneva: World Health Organization; 2015 [cited 2019 Dec 18]. Available from: http://www.stoptb.org/global/plan/plan2/

2. Ministério da Saúde (BR). Brasil Livre da Tuberculose: evolução dos cenários epidemiológicos e operacionais da doença. Boletim Epidemiológico. 2019;50(9):1-18.

3. Ministério da Saúde (BR), Secretaria de Vigilância em Saúde. Brasil livre da tuberculose: evolução dos cenários epidemiológicos e operacionais da doença. Boletim Epidemiológico. 2019;50(9):1-18.

4. Ministério da Saúde (BR), Secretaria de Vigilância em Saúde, Departamento de Vigilância das Doenças Transmissíveis. Brasil Livre da Tuberculose: plano nacional pelo fim da tuberculose como problema de saúde pública. Brasília (DF): Ministério da Saúde; 2017.

5. Ministério da Saúde (BR), Secretaria de Vigilância em Saúde, Departamento de Vigilância das Doenças Transmissíveis. Manual de recomendações para o controle da tuberculose no Brasil. Brasília (DF): Ministério da Saúde; 2018.

6. Sacramento DS, Lavor DCBS, Oliveira LRT, Gomes APBL, Gonçalves MJF. Organização dos serviços de saúde para o diagnóstico e tratamento dos casos de tuberculose em Manaus, Amazonas, 2014. Epidemiol Serv Saúde (Online). 2019;28(2):e2017500. doi: https://doi.org/10.5123/S1679-49742019000200007 
7. Peri AM, Bernasconi DP, Galizzi N, Matteelli A, Codecasa L, Giorgio V, et al. Determinants of patient and health care services delays for tuberculosis diagnosis in Italy: a cross-sectional observational study. BMC Infect Dis. 2018;18(1):690. doi: https://doi.org/10.1186/s12879-018-3609-4

8. Bogale S, Diro E, Shiferaw AM, Yenit MK. Factors associated with the length of delay with tuberculosis diagnosis and treatment among adult tuberculosis patients attending at public health facilities in Gondar town, Northwest, Ethiopia. BMC Infect Dis. 2017;17(1):145. doi: https://doi.org/10.1186/s12879-017-2240-0

9. Spagnolo LML, Tomberg JO, Vieira DA, Gonzales RIC. Detection of tuberculosis: respiratory symptoms flow and results achieved. Rev Bras Enferm. 2018;71(5):2543-51. doi: http://dx.doi.org/10.1590/0034-7167-2017-0457

10. Gebreegziabher SB, Bjune GA, Yimer SA. Patients' and health system's delays in the diagnosis and treatment of new pulmonar tuberculosis patients in West Gojjam Zone, Northwest Ethiopia: a crosssectional study. BMC Infect Dis. 2016;16(11):673. doi: https://doi.org/10.1186/s12879-016-1995-z

11. Cecílio HPM, Teston EF, Marcon SS. Access to the diagnosis of tuberculosis from the point of view of health professionals. Texto \& Contexto Enferm. 2017;26(3):e0230014. doi: https://doi.org/10.1590/010407072017000230014

12. Gonzales, Roxana Isabel Cardozo. Atenção primária à saúde na detecção de casos de tuberculose em municípios prioritários do sul do Brasil: desafios e investimentos em estratégias de informação. Chamada MCTI/CNPq/MS-SCTIE - Decit N 40/2012. 2012. Resumo em: https://institucional.ufpel.edu.br/projetos/id/p6794

13. Secretaria Estadual de Saúde (RS), Centro Estadual de Vigilância em Saúde, Divisão de Vigilância Epidemiológica. Programa Estadual de Controle da Tuberculose (PECT/RS). Informe epidemiológico: tuberculose. Porto Alegre (RS): Secretaria Estadual de Saúde; 2018.

14. BRASIL. Ministério da Saúde. Resolução no 466, de 12 de dezembro de 2012. Divulga as diretrizes e normas regulamentadoras das pesquisas e testes em seres humanos. Diário Oficial da União: Seção 1, Brasília, DF, n. 112, p. 59, 13 jun. 2013.

15. Sá LD, Scatena LM, Rodrigues RAP, Nogueira JA, Silva AO, Villa TCS. Gateway to the diagnosis of tuberculosis among elders in Brazilian municipalities. Rev Bras Enferm. 2015;68(3):408-14. doi: http://dx.doi.org/10.1590/0034-7167.2015680313i

16. Bartholomay P, Pelissari DM, Araujo WN, Yadon ZE, Heldal E. Quality of tuberculosis care at different levels of health care in Brazil in 2013. Rev Panam Salud Publica. 2016;39(1):3-11.

17. Grut L, Sanudi L, Braathen SH, Jürgens T, Eide AH. Access to tuberculosis services for individuals with disability in rural Malawi, a qualitative study. PLoS One. 2015;10(4):e0122748. doi: https://doi.org/10.1371/journal.pone.0122748 
18. Pinheiro PGOD, Sá LD, Palha PF, Oliveira RCO, Nogueira JA, Villa TCS. Critical points for the control of Tuberculosis on Primary Health Care. Rev Bras Enferm. 2017;70(6):1227-34. doi: https://doi.org/10.1590/0034-7167-2016-0467

19. Stošić M, Lazarević N, Kuruc V, Ristić L. Assessment of the role of primary health care in tuberculosis control in Serbia. Med Pregl. 2015;9(10):331-5. doi: https://doi.org/10.2298/MPNS1510331S

20. Ponce MAZ, Wysocki AD, Arakawa T, Andrade RLP, Vendramini SHF, Silva Sobrinho RA, et al. Atraso do diagnóstico da tuberculose em adultos em um município paulista em 2009: estudo transversal. Epidemiol Serv Saúde [Internet]. 2016 [acesso em 2020 jan 03]; 25(3):553-62. Disponível em: https://www.scielo.br/pdf/ress/v25n3/2237-9622-ress-25-03-00553.pdf

\section{Autor correspondente}

Jéssica Oliveira Tomberg

E-mail: jessicatomberg@hotmail.com

Endreço: Rua Gomes Carneiro, 647

CEP: 96010-610, Pelotas, RS, Brasil

\section{Contribuições de Autoria}

\section{1 - Jéssica Oliveira Tomberg}

contribuição substancial para a concepção do estudo, coleta de dados, análise estatística, conceitualização; elaboração e revisão crítica do conteúdo intelectual; metodologia; preparo do original, redação e edição; supervisão; aprovação da versão final do estudo a ser publicado.

\section{2 - Lílian Moura de Lima Spagnolo}

contribuição substancial para a concepção do estudo, coleta de dados, análise estatística, conceitualização, elaboração e revisão crítica do conteúdo intelectual, metodologia, redação e edição, aprovação da versão final do estudo a ser publicado.

\section{3 - Jenifer Harter}

coleta de dados, análise estatística, conceitualização, elaboração e revisão crítica do conteúdo intelectual, aprovação da versão final do estudo a ser publicado.

\section{4 - Martina Dias da Rosa Martins}

contribuição substancial para a concepção do estudo, análise estatística, redação e edição, aprovação da versão final do estudo a ser publicado.

\section{5 - Roxana Isabel Cardozo Gonzales}

contribuição substancial para a concepção do estudo; aquisição de financiamento; gerenciamento de financiamento; gerenciamento do projeto; coleta de dados; elaboração e revisão crítica do conteúdo intelectual; metodologia; supervisão; aprovação da versão final do estudo a ser publicado. 
Comportamento de busca por serviços de saúde para a detecção da tuberculose | 18

\section{Como citar este artigo}

Tomberg JO, Spagnolo LML, Härter J, Martins MDR, Gonzales RIC. Comportamento de busca por serviços de saúde para a detecção da tuberculose. Rev. Enferm. UFSM. 2020 [Acesso em: Anos Mês Dia]; vol.10 e52: 1-18. DOI:https://doi.org/10.5902/2179769241815 